\title{
Thermal Simulation for Two-Phase Liquid Cooling 3D-ICs
}

\author{
Hong-Wen Chiou' ${ }^{1,2}$, Yu-Min Lee ${ }^{1}$ \\ ${ }^{1}$ Department of Electrical and Computer Engineering, National Chiao Tung University, Hsinchu, Chinese Taipei \\ ${ }^{2}$ Industrial Technology Research Institute, Hsinchu, Chinese Taipei \\ Email: simon00195.cm01g@nctu.edu.tw, yumin@nctu.edu.tw
}

How to cite this paper: Chiou, H.-W. and Lee, Y.-M. (2016) Thermal Simulation for Two-Phase Liquid Cooling 3D-ICs. Journai of Computer and Communications, 4, 3345 .

http://dx.doi.org/10.4236/jcc.2016.415003

Received: September 12, 2016 Accepted: November 25, 2016 Published: November 28, 2016

\begin{abstract}
This work presents an algorithm for simulating more accurate temperature distribution in two-phase liquid cooling for three-dimensional integrated circuits than the state of-the-art methods by utilizing local multi-linear interpolation techniques on heat transfer coefficients between the microchannel and silicon substrate, and considering the interdependence between the thermal conductivity of silicon and temperature values. The experimental results show that the maximum and average errors are only $9.7 \%$ and $6.7 \%$ compared with the measurements, respectively.
\end{abstract}

\section{Keywords}

Thermal Simulation, Microchannel, Two-Phase Liquid Cooling

\section{Introduction}

Due to the high power density and the ill of heat dissipation capability, the operating temperature of three-dimensional integrated circuits (3-D ICs) is higher than that of two-dimensional (2-D) ICs. Recently, to effectively remove the heat in 3-D ICs, the advanced liquid cooling system has been widely discussed [1]. Different from the conventional air cooling system, the liquid cooling system delivers water or refrigerant to microchannels heat sink. The microchannels are etched in the silicon substrate in advance. As pointed out in Ref. [2], the chip, which integrates microchannels, can consume a very high power density $\left(790 \mathrm{~W} / \mathrm{cm}^{2}\right)$ without burning down. Reference [3] discretized microchannels into small grids to propose a steady state thermal model for single-phase liquid cooling 3-D ICs. Four extra nodes are added, and a channel merging technique is used to simulate the complicated thermal-wake effect of liquid cooling. In addition, 3D-ICE, a compact transient thermal model was proposed in Ref. [4]. The convection heat transfer that leads to thermal-wake effect is already considered in 3D-ICE, so no 
extra nodes are required. Based on 3D-ICE, other works use a similar model but develop different system solving methods such as the neural network [5], the operator splitting [6], and the explicit method [7].

Different from the above single-phase liquid cooling systems, the two-phase liquid cooling removes the heat via heat absorption during the evaporation process [8]. After absorbing the heat, the part of refrigerant changes its physical state from liquid to vapor. Therefore, qualifying the fraction of vapor becomes an important issue. Since the heat transfer coefficients (HTCs) at the wall surface between solids and liquids depend on the physical state of refrigerant and the temperature profile of chip, an iterative method, STEAM, was proposed to iteratively solve the steady-state problem [8].

This work provides an accurate temperature profile estimate for the two-phase liquid cooling system of 3-D IC. The inputs are the design structure and material files, and the power distribution in device layers. Different from incorporating the fitting curves for correlating HTCs at the wall surface in STEAM [8], here, we present a multi-linear interpolation method on measurement database of HTCs obtained from [9]. Moreover, the proposed flow considers the change of the thermal conductivities of silicon varying from temperature values since there are appreciable temperature gradients between device layers and microchannel layers. Finally, the output is the temperature profile of design. The main contributions of this work are

- By using a local multi-linear interpolation technique with the measurement database to approximate HTCs during the thermal simulation procedure for the two-phase liquid cooling system, the accuracy can be reasonably improved.

- Considering the interdependence between the operating temperature and thermal conductivity of silicon does improve the simulation accuracy for two-phase liquid cooling systems.

The remainder of this paper is organized as follows. Section 2 reviews the thermal model for at wophase liquid-cooled 3-D IC. With the thermal model, the proposed thermal simulation algorithm is detailed in Section 3. The experimental results are presented in Section 4, and finally, conclusions are drawn in Section 5.

\section{Preliminary}

The structure of a liquid-cooled 3-D IC is shown in Figure 1. Interconnect layers contain

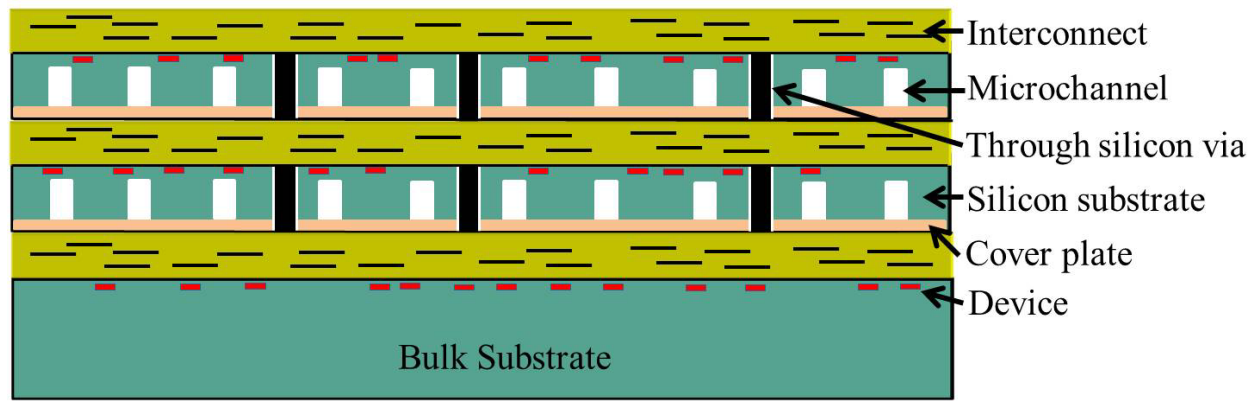

Figure 1. Thermal model of a liquid-cooled 3-D IC with three tiers. 
interconnects and dielectric. Each device layer is a thin layer on the top surface of stacked silicon substrate or silicon bulk. Functional blocks of each tier are modeled as power generating sources attached to the thin layer that is close to the top surface of the stacked silicon substrateor the silicon bulk. Microchannels are etched on the silicon substrate as heat sink, and a cover plate layer is placed on the back-side of silicon substrate. The material of cover plate is either Si or Pyrex glass. Through silicon vias are placed in the silicon substrate and cover plate layer. All the boundary surfaces of a 3-D IC are assumed to be adiabatic as boundary conditions.

According to the heat transfer equation, the temperature profile $T(\mathbf{r})$ in solids can be governed by

$$
\nabla \cdot[\kappa(\mathbf{r}, T) \nabla T(\mathbf{r})]=-p(\mathbf{r}),
$$

and $\nabla T(\mathbf{r})$ in fluids (liquid and vapor) can be governed by

$$
\nabla \cdot[\kappa(\mathbf{r}, T) \nabla T(\mathbf{r})]-c_{v} \vec{u} \cdot \nabla T(\mathbf{r})=-p(\mathbf{r}),
$$

Here, $\mathbf{r}=(x, y, z), \kappa(\mathbf{r}, T)$ is the thermal conductivity at $\mathbf{r}, c_{v}$ is the volumetric specific heat, $p(\mathbf{r})$ is the power density profile of chip, and $\vec{u}$ is the velocity of outflow at the surface of the control volume.

\subsection{Thermal Model of Solid Grids}

Using the finite difference method and the duality between thermal and electrical quantities, (1) can be transformed to a SPICE compatible equivalent thermal circuit containing thermal resistances and power sources. For a specific control volume (thermal grid) as shown in Figure 2, each thermal conductance can be calculated as

$$
g_{(i, j, k) \leftrightarrow(i \pm 1, j, k)}=\kappa \frac{2 w l}{h} ; g_{(i, j, k) \leftrightarrow(i, j \pm 1, k t)}=\kappa \frac{2 l h}{w} ; g_{(i, j, k) \leftrightarrow(i, j, k \pm 1)}=\kappa \frac{2 w h}{l} .
$$

Here, $g_{(i, j, k) \leftrightarrow\left(i^{\prime}, j^{\prime}, k^{\prime}\right)}$ is the thermal conductance between grid $(i, j, k)$ and its neighboring grid $\left(i^{\prime}, j^{\prime}, k^{\prime}\right) . l, w$, and $h$ are the length, width, and height of grid $(i, j, k)$, respectively.

The temperature $T i, j, k$ at the central node of each solid grid $V i, j, k$ need to satisfy the following modified nodal analysis (MNA) system.

$$
\mathbf{G}_{\mathrm{s}}(\mathbf{T}) \mathbf{T}=\mathbf{P},
$$

Here, $\mathbf{G}_{\mathrm{s}}(\mathbf{T})$ is an augmented temperature-dependent thermal conductance matrix (contains thermal conductance in solids and HTCs between solids and fluids), $\mathbf{T}$ is a temperature profile vector of thermal grids, and $\mathbf{P}$ is the power profile vector in solids.

\subsection{Thermal Model of Two-Phase Grids [8]}

Given a thermal grid in liquids as shown in Figure 3, by using the law of energy and mass conservation, the energy balance equation can be written as follows from STEAM [8] 

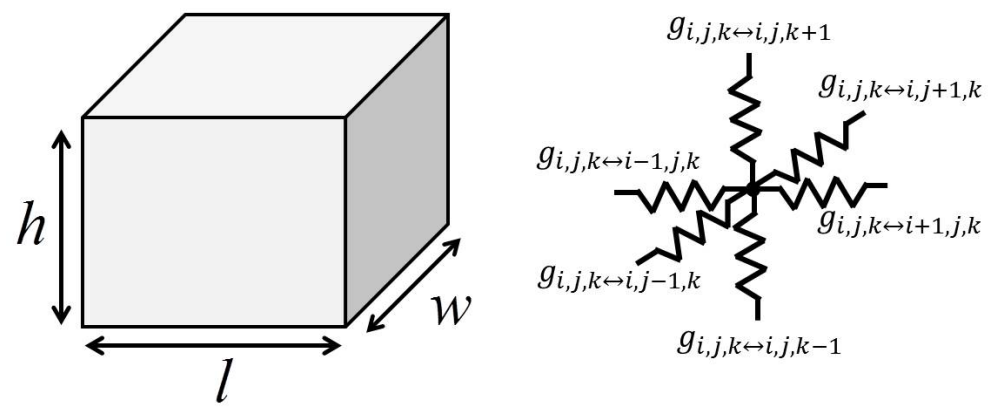

Figure 2. Equivalent circuit of a solid thermal grid.

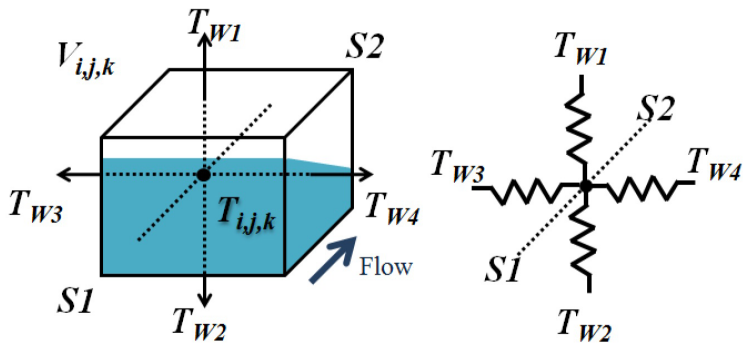

Figure 3. Two-phase thermal grid [8].

$$
h_{l v} \dot{M} \chi_{S 1}+\sum_{s=1}^{4} \alpha_{W}\left(T_{W S}-T_{i, j, k}\right)=h_{l v} \dot{M} \chi_{S 2}
$$

Here, $T_{i, j, k}$ is the central temperature at refrigerant thermal grid $V_{i, j, k}, h_{l v}$ is the latent heat of vaporization, $\dot{M}$ is the mass flowrate, $T_{W S}$ is the temperature on the wall of thermal grid, $\chi_{S 1} / \chi_{S 2}$ is refrigerant vapor quality at the interface $S 1 / S 2$, and $\alpha_{W}$ is the heat transfer coefficient at the wall surface.

In (4), the second term on left-hand side represents the heat entering from the microchannel sidewalls into each two-phase thermal grid, and other terms represent heat removal denoted by the latent heat difference of vaporization between the two interfaces $S 1$ and $S 2 . \alpha_{W}$ is correlated with the mass flux $j_{\mathrm{m}}$, vapor quality $\chi_{i, j, k}$, heat flux density $q^{\prime \prime}$ from solid into two-phase liquid, diameter of microchannels $D_{\mathrm{h}}$, Prandtl number $\operatorname{Pr}$, and the confinement number Co in different situation. Several nonlinear fitting curves were applied to fit the measurement data for constructing the HTC correlation functions in [9] [10] [11]. Since $\alpha_{W}$ relies on multiple parameters, it is hard to find a suitable fitting form globally, and hence, the existing fitting forms could lead to larger errors in some local regions. Moreover, heat flux $q$ " and vapor quality $\chi_{i, j, k}$ are still unknown so as to solving heat transfer equation iteratively. As a result, we propose to use a local multi-linear interpolation technique with the measurement database to approximate $\alpha_{W}$ in each iteration and grids.

\section{Proposed Method}

Based on a similar approach as STEAM [8], which the refrigerant temperature values are assumed to be known (they are iteratively calculated during the solving procedure.), 
(3) and (4) can be combined to build the heat transfer equation for a two-phase liquidcooled IC as

$$
\mathbf{G}(\mathbf{T}) \mathbf{X}=\mathbf{U} .
$$

Here, $\mathbf{G}(\mathbf{T})$ is a temperature-dependent thermal conductance matrix, $\mathbf{X}$ is the temperature and vapor quality vector of grids, and $\mathbf{U}$ is the source and boundary condition vector.

The flow chart of our proposed algorithm for solving the vector $\mathbf{X}$ in (5) is shown in Figure 4.

1) In the beginning, the thermal conductivities and power densities are stamped into the matrix. Since HTCs in two-phase liquid grids are unknown, based on the inlet condition of refrigerant, these unknown parameters are initially guessed.

2) After the MNA system is established, Super LU solver [12] is utilized to solve (5). At this iteration, the temperature profile $\mathbf{T}$ and vapor quality $\chi$ are renewed.

3) With the temperature values adjacent to the two-phase liquid grids and HTCs, each heat flux density $q$ " can be computed. After that, HTCs can be updated by using the multi-linear interpolation technique with six parameters (variables) described in Section 3.1 .

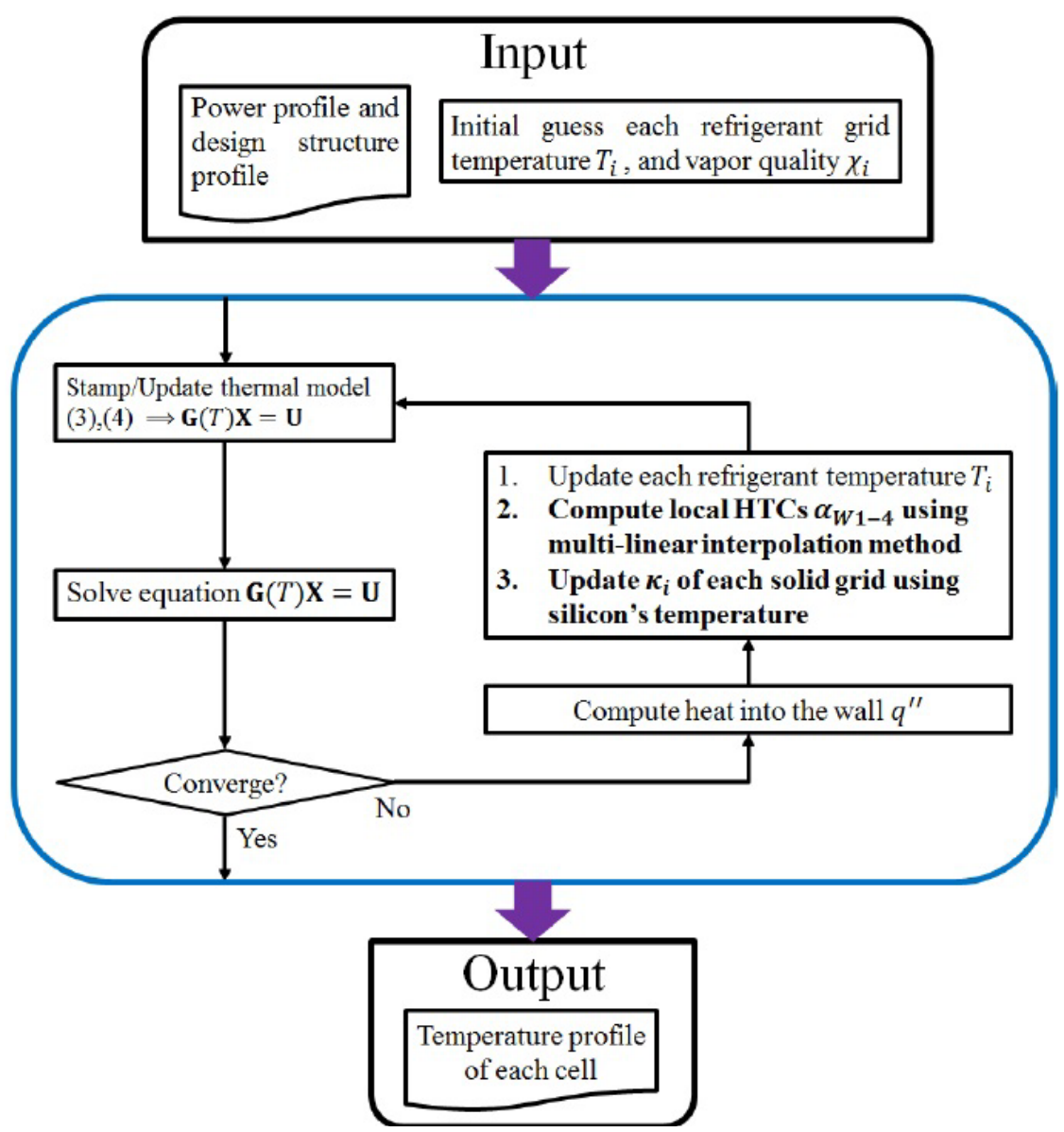

Figure 4. Flow chart of the proposed algorithm. 
4) With the temperature values at solid grids of silicon, the temperature dependent thermal conductance $\kappa_{i}$ a teach solid grid of silicon is updated as described in Section 3.2.

5) By using the homogeneous equation for pressure drop [13], the pressure of each two-phase liquid grid can be computed. By using REFPROP [14], the refrigerant temperature $T_{i}$ of each liquid grid $i$ can be obtained.

6) The results obtained by 3 )-5) are sent back to 2 ), and the whole procedure is repeated until $\mathbf{X}$ is convergent.

\subsection{Multi-Linear Interpolation Method for Calculating Heat Transfer Coefficients}

1) Pre-build a regular table of HTCs by the interpolation method on irregular measurement data points. Directly using the measurement data points of HTCs (3899 data points) [9] to perform the interpolation for updating each HTC during the simulation flow is hard and inefficient, since the distribution of measured points is irregular and the HTC is correlated with six parameters. Therefore, we first utilize those measurement data to pre-build a regular table of HTCs.

To simply illustrate the pre-building procedure, we assume that the measurement data $\left(f_{m_{i}}\right)_{i=1}^{n}$ are correlated with two parameters $\left(\left(u_{m_{i}}, v_{m_{i}}\right)\right)_{i=1}^{n}$, and each $f_{m_{i}}$ is measured at $\left(u_{m_{i}}, v_{m_{i}}\right)$. In the beginning, as shown in Figure 5, these two parameters are normalized for balancing the relation between them. Next, the desired points of the regular table are decided. After that, the normalized measured-point region is uniformly partitioned into many sub-regions. Each desired point belongs to a sub-region, and its artificial measurement is interpolated by those points inside that sub-region. The inverse distance weighting method [15] that is suitable for the irregular measured points is chosen to build the table. For example, as shown in Figure 5, $\left(u_{4}, v_{2}\right)$ is the desired point, and its value $F_{\mathrm{t}\left(u_{4}, v_{2}\right)}$ can be interpolated as follows.

$$
F_{\mathrm{t}\left(u_{4}, v_{2}\right)}=\frac{\sum_{\left(u_{m_{i}}, v_{m_{i}}\right) \in \mathcal{S}_{u, v}}\left(d_{m_{i}}^{-1}-d^{-1}\right)^{2} f_{m_{i}}}{\sum_{\left(u_{m_{j}}, v_{m_{j}}\right) \in \mathcal{S}_{u, v}}\left(d_{m_{j}}^{-1}-d^{-1}\right)^{2}}
$$

where $d_{m_{i}}=\sqrt{\left(u-u_{m_{i}}\right)^{2}+\left(v-v_{m_{i}}\right)^{2}}$, and $d=\max _{\left(u_{m_{i}}, v_{m_{i}}\right) \in \mathcal{S}_{u, v}} d_{m_{i}}$.

The interpolation form (6) indicates that the more the measured point is close to the desired point, the more its influence is. For building the regular HTC table, the formula is presented as follows.

$$
H T C_{\mathrm{t}(\mathbf{e})}=\frac{\sum_{\mathbf{e}_{m_{i}} \in \mathcal{S}_{\mathbf{e}}}\left(d_{m_{i}}^{-1}-d^{-1}\right)^{2} \operatorname{HTC}\left(\mathbf{e}_{m_{i}}\right)}{\sum_{\mathbf{e}_{m_{j}} \in \mathcal{S}_{\mathbf{e}}}\left(d_{m_{j}}^{-1}-d^{-1}\right)^{2}}
$$

Here, $\operatorname{HTC}\left(\mathbf{e}_{m_{i}}\right)$ is the given measurement of HTCat $\mathbf{e}_{m_{i}}$, $\mathbf{e}=\left(q^{\prime \prime}, \chi, D_{\mathrm{h}}, \operatorname{Pr}, C o, j_{\mathrm{m}}\right), \mathbf{e}_{m_{i}}=\left(q_{m_{i}}^{\prime \prime}, \chi_{m_{i}}, D_{\mathrm{h}_{i}}, \operatorname{Pr}_{m_{i}}, C o_{m_{i}}, j_{\mathrm{m} m_{i}}\right), d_{m_{i}}$ is the Euclidean distance between $\mathbf{e}_{m_{i}}$ and $\mathbf{e}$, and $d=\max _{\mathbf{e}_{m_{i}} \in \mathcal{S}_{\mathbf{e}}} d_{m_{i}}$. 
2) Calculate HTCs by the multi-linear interpolation method on regular points at built HTC table: Utilizing the built regular HTC table, HTCs can be efficiently calculated by the multi-linear interpolation technique during the simulation procedure. There are many ways for solving interpolation method on regular grids, such as bilinear interpolation, bicubic interpolation, spline interpolation, etc. Here, the multi-linear interpolation on six variables is adopted.

Again, for simplicity, the same example shown in Figure $\mathbf{5}$ is used to illustrate the interpolation procedure. The value $F_{(u, v)}$ at point $(u, v)$ shown in Figure 5 can be interpolated as

$$
\begin{aligned}
F_{(u, v)}=F_{\mathrm{t}\left(u_{1}, v_{1}\right)} & \frac{\left(u_{2}-u\right)\left(v_{2}-v\right)}{\left(u_{2}-u_{1}\right)\left(v_{2}-v_{1}\right)}+F_{\mathrm{t}\left(u_{2}, v_{1}\right)} \frac{\left(u-u_{1}\right)\left(v_{2}-v\right)}{\left(u_{2}-u_{1}\right)\left(v_{2}-v_{1}\right)} \\
& +F_{\mathrm{t}\left(u_{1}, v_{2}\right)} \frac{\left(u_{2}-u\right)\left(v-v_{1}\right)}{\left(u_{2}-u_{1}\right)\left(v_{2}-v_{1}\right)}+F_{\mathrm{t}\left(u_{2}, v_{2}\right)} \frac{\left(u-u_{1}\right)\left(v-v_{1}\right)}{\left(u_{2}-u_{1}\right)\left(v_{2}-v_{1}\right)} .
\end{aligned}
$$

Here, $u_{1}<u<u_{2}, v_{1}<v<v_{2}$, and $\left(u_{1}, v_{1}\right),\left(u_{2}, v_{1}\right),\left(u_{1}, v_{2}\right)$ and $\left(u_{2}, v_{2}\right)$ form a regular grid.

Therefore, in each iteration, the HTC at an arbitrary point e can be calculated by using the above multi-linear interpolation method with six parameters as

$$
\begin{aligned}
H T C_{(\mathbf{e})}= & N_{\mathbf{e}}^{1} \cdot H T C_{\mathrm{t}\left(\chi_{1}, q_{1}^{\prime \prime}, C \sigma_{1}, D_{\mathrm{h} 1}, \mathrm{Pr}_{1}, j_{\mathrm{m} 1}\right)}+N_{\mathbf{e}}^{2} \cdot H T C_{\mathrm{t}\left(\chi_{1}, q_{2}, C \sigma_{1}, D_{\mathrm{h} 1}, \mathrm{P}_{1}, j_{\mathrm{m} 1}\right)}+\cdots \\
& +N_{\mathbf{e}}^{64} \cdot H T C_{\mathrm{t}\left(\chi_{2}, q_{2}^{\prime \prime}, C_{2}, D_{\mathrm{h} 2}, \mathrm{Pr}_{2}, j_{\mathrm{m} 2}\right)},
\end{aligned}
$$

where each $H T C_{\mathrm{t}\left(\chi_{1 \mid 1}, q_{12}^{\prime \prime}, C_{112}, \mathrm{P}_{\mathrm{h} \mid 2}, \mathrm{P}_{\mathrm{F}_{12},}, j_{\mathrm{m} \mid 12}\right)}$ is pre-established by $(7)$, and each $N_{\mathbf{e}}^{i}$ $(i=1 \sim 64)$ is equal to

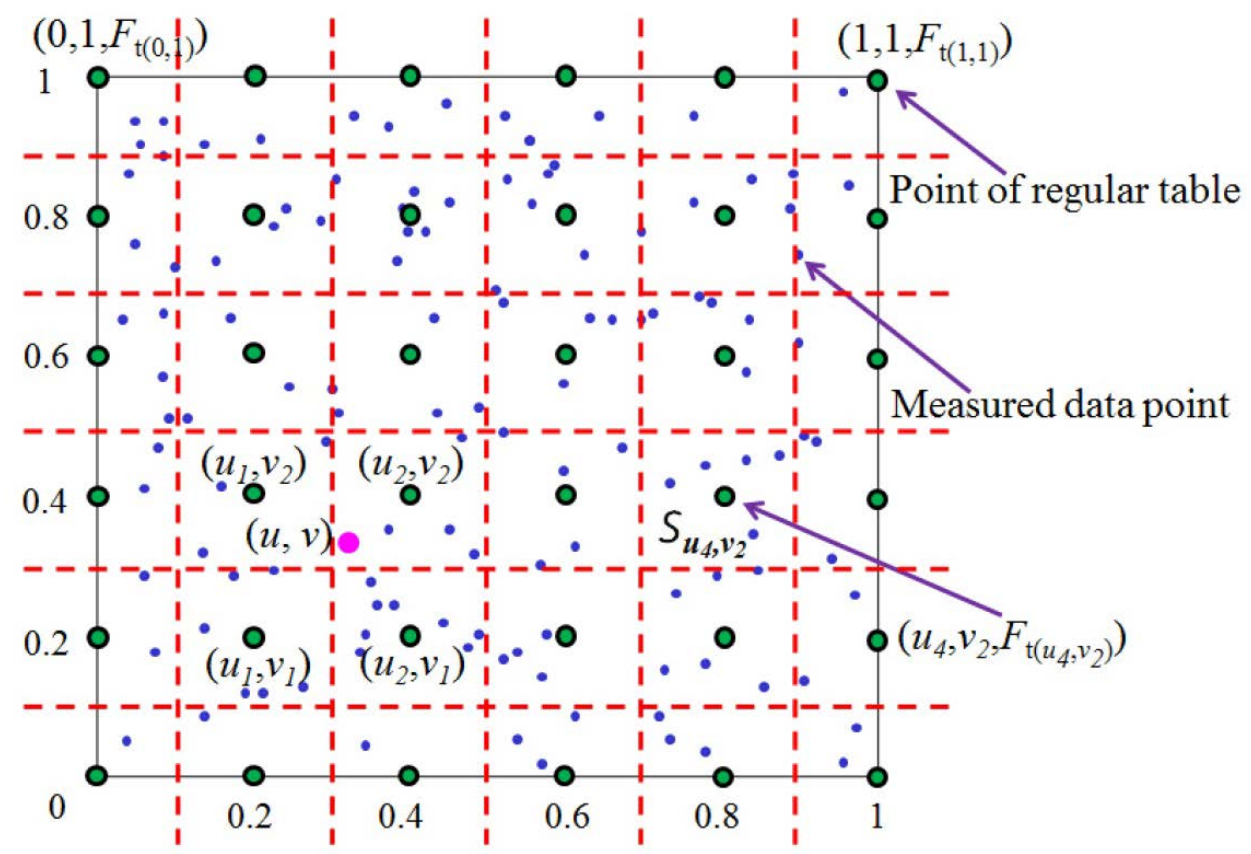

Figure 5. Illustration of the interpolation method. 


$$
\begin{aligned}
N_{\mathrm{e}}^{1}= & \frac{\left(\chi_{2}-\chi\right)\left(q_{2}^{\prime \prime}-q^{\prime \prime}\right) \cdots\left(j_{\mathrm{m} 2}-j_{\mathrm{m}}\right)}{\left(\chi_{2}-\chi_{1}\right)\left(q_{2}^{\prime \prime}-q_{1}^{\prime \prime}\right) \cdots\left(j_{\mathrm{m} 2}-j_{\mathrm{m} 1}\right)}, \\
N_{\mathrm{e}}^{2}= & \frac{\left(\chi_{2}-\chi\right)\left(q^{\prime \prime}-q_{1}^{\prime \prime}\right) \cdots\left(j_{\mathrm{m} 2}-j_{\mathrm{m}}\right)}{\left(\chi_{2}-\chi_{1}\right)\left(q_{2}^{\prime \prime}-q_{1}^{\prime \prime}\right) \cdots\left(j_{\mathrm{m} 2}-j_{\mathrm{m} 1}\right)}, \\
& \vdots \\
N_{\mathrm{e}}^{64} & =\frac{\left(\chi-\chi_{1}\right)\left(q^{\prime \prime}-q_{1}^{\prime \prime}\right) \cdots\left(j_{\mathrm{m}}-j_{\mathrm{m} 1}\right)}{\left(\chi_{2}-\chi_{1}\right)\left(q_{2}^{\prime \prime}-q_{1}^{\prime \prime}\right) \cdots\left(j_{\mathrm{m} 2}-j_{\mathrm{m} 1}\right)} .
\end{aligned}
$$

\subsection{Temperature Dependent Thermal Conductivity of Silicon}

For air-cooling or single-phase liquid cooling 3-D ICs, the gradient of temperature along $\mathrm{z}$-axis (normal to the device surface) could be low. However, the refrigerant temperature is almost unchanged (in the situation, the liquid is not dried out), so the gradient of temperature along $\mathrm{z}$-axis might be high in some cases. As a result, the temperature-dependent thermal conductivity should be considered. In this work, the formula of thermal conductivity versus temperature presented in [16] is used to update the thermal conductivity for the solid thermal grid.

$$
\kappa(T)=\kappa\left(T_{0}\right)\left(\frac{T}{T_{0}}\right)^{-\beta}
$$

Here, $\kappa\left(T_{0}\right)$ is the thermal conductivity at temperature $T_{0}=300 K, \kappa(T)$ is the thermal conductivity at temperature $T\left(T \geq T_{0}\right)$, and $\beta \approx 1.324$ for silicon.

\subsection{Transient Thermal Simulation}

By applying the backward Euler method into (1) and (2), we have

$$
\mathbf{G T}_{i}+\frac{1}{\Delta t}\left(\mathbf{C}_{i} \mathbf{T}_{i}-\mathbf{C}_{i-1} \mathbf{T}_{i-1}\right)=\mathbf{P}_{i},
$$

where $\Delta t$ is the constant time step, $\mathbf{C}_{i}$ and $\mathbf{C}_{i-1}$ are heat capacitance matrix at time step $i$ and $i-1, \mathbf{T}_{i}$ and $\mathbf{T}_{i-1}$ are the temperature distribution at time step $i$ and $i-1$, respectively. $\mathbf{P}_{i}$ is the power vector for representing equivalent power values of the control volumes at time step $i$. Since the decomposition of capacitance in two-phase grids may be changed during each iteration, re-performing LU decomposition of system matrix is needed, and the flow chart is shown in Figure 6.

\section{Experimental Results}

The proposed method is implemented in $\mathrm{C}++$ language and tested on Intel Processor i7-3770 3.40 GHz CPU with 20GB RAM. Three pseudo chips with their measured data obtained by [17] are used to verify the proposed method. Meanwhile, ANSYS CFD [18], which is used to verify the simulation results, is used to verify the proposed method as well.

The structure of pseudo chips is shown in Figure 7. The thermal conductivity $(\mathrm{W} / \mathrm{m} \cdot \mathrm{K})$ of silicon, cover plate, and TIM are 148, 1.4, and 22 at $300 \mathrm{~K}$, respectively. The 


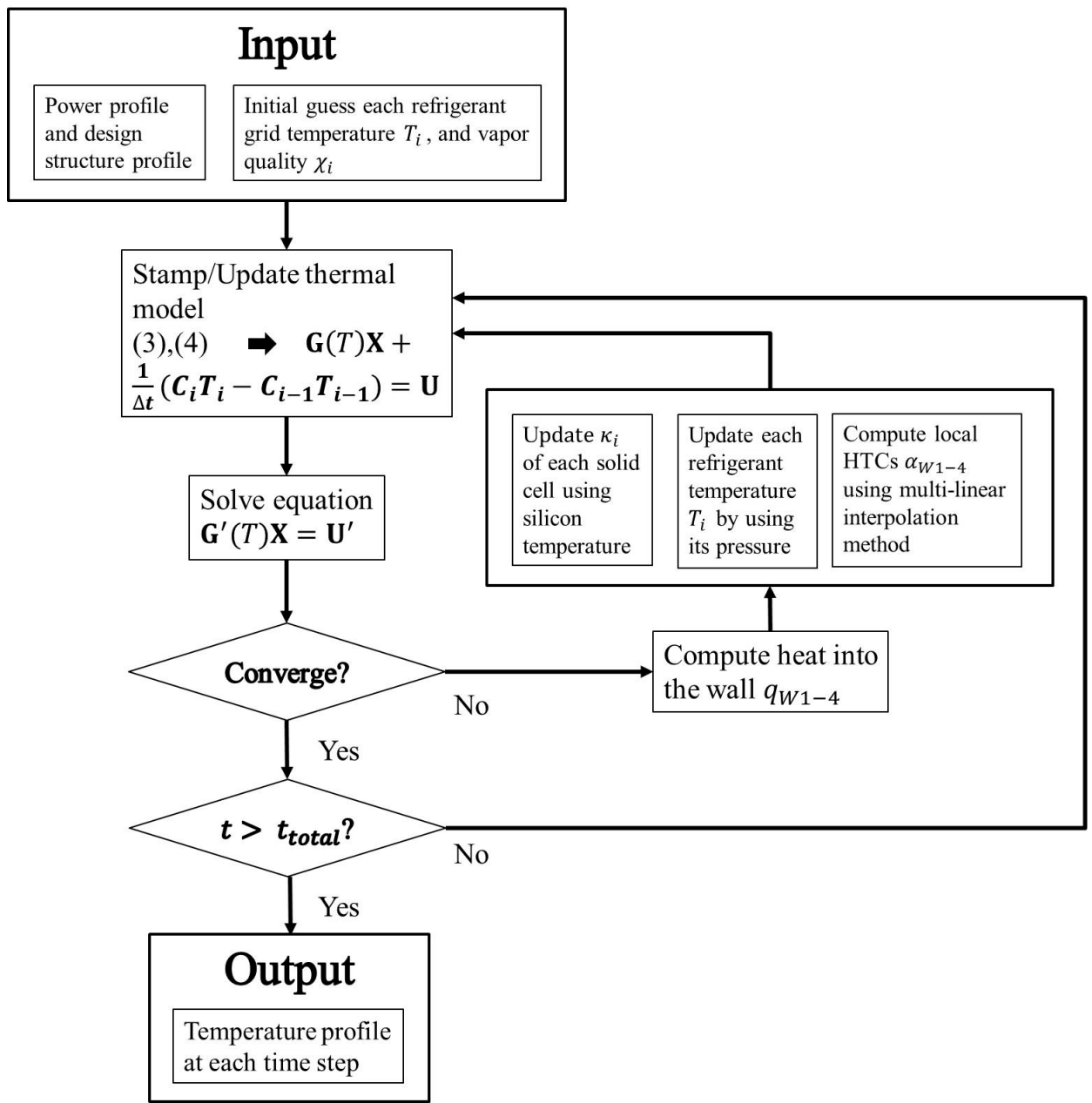

Figure 6. Flow chart of the proposed algorithm for transient simulation.

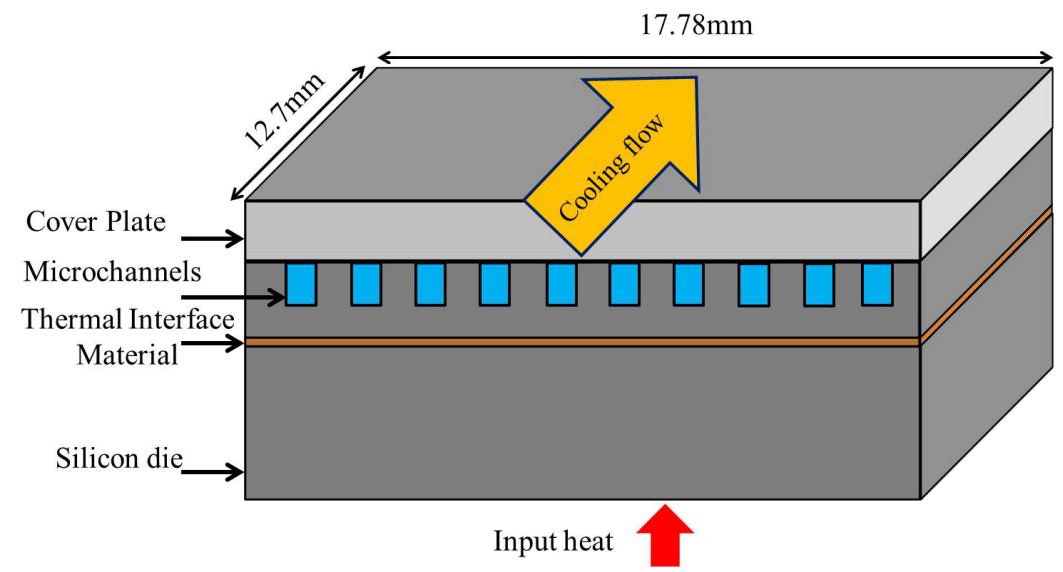

Figure 7. The geometry structure of pseudo chips.

chosen refrigerant is R236-fa. Their power maps are shown in Figures 8(a)-(c). Figure 8(a) presents the uniform power map case, its power density is $96.4 \mathrm{~W} / \mathrm{cm}^{2}$, the inlet temperature is $304.1 \mathrm{~K}$, and the mass flux is $933 \mathrm{~kg} / \mathrm{m}^{2} \mathrm{~s}$. For the point hotspot power 


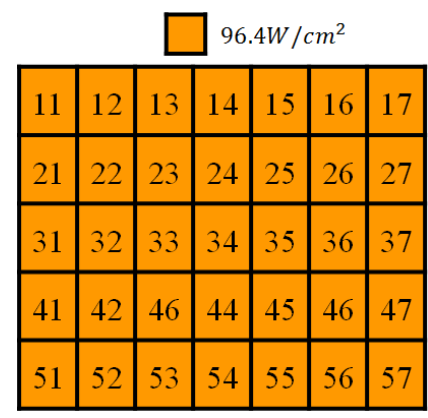

(a)

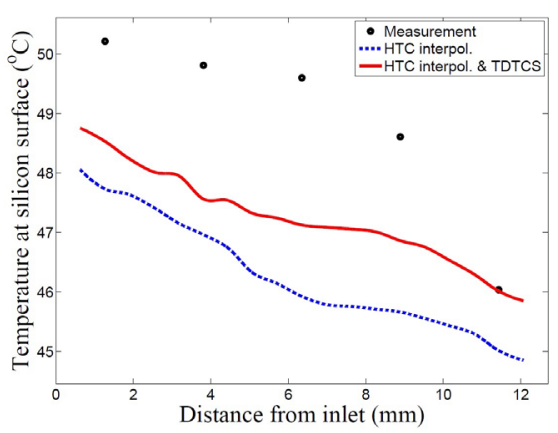

(d)

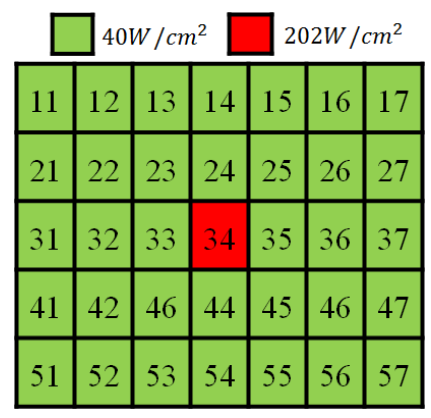

(b)

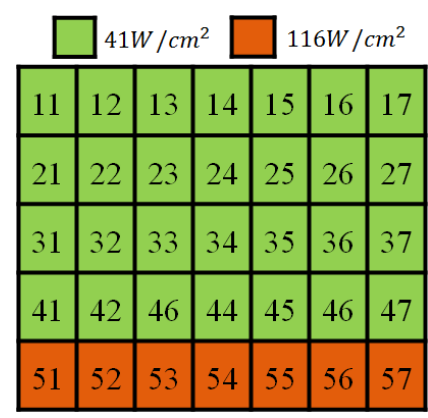

(c)

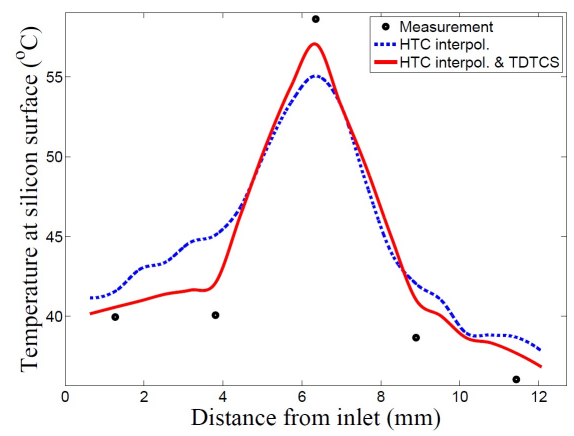

(e)

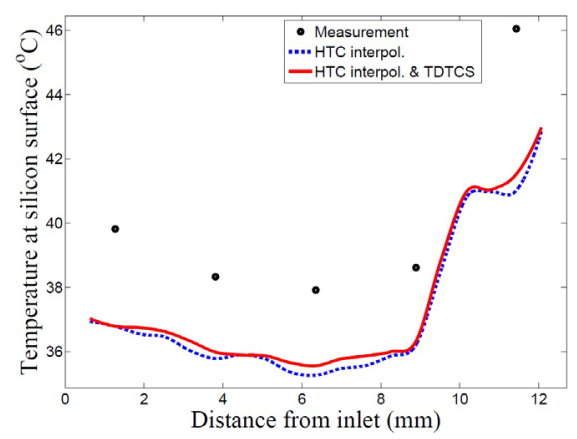

(f)

Figure 8. (a)-(c) Power map; (d)-(f) Temperature comparison of col\#4 between the proposed method and measured data. (a) Uniform power map; (b) Point hotspot power map; (c) Row hotspot power map; (d) Temperatures of uniform power map; (e) Temperatures of point hotspot power map; (f) Temperatures of row hotspot power map.

map case shown in Figure 8(b), its power density is $40 \mathrm{~W} / \mathrm{cm}^{2}$ except that the grid 34 is $202 \mathrm{~W} / \mathrm{cm}^{2}$. Its inlet temperature is $304.1 \mathrm{~K}$, and mass flux is $713 \mathrm{~kg} / \mathrm{m}^{2} \mathrm{~s}$. The power density of row hotspot power map as shown in Figure $8(\mathrm{c})$ is $41 \mathrm{~W} / \mathrm{cm}^{2}$ from row\#1 to row\#4, and $116 \mathrm{~W} / \mathrm{cm}^{2}$ in row\#5 (grids 51 - 57). Its inlet temperature is $303.5 \mathrm{~K}$, and mass flux is $895 \mathrm{~kg} / \mathrm{m}^{2} \mathrm{~s}$.

The temperature comparison of col\#4 between the proposed method and the measured data is shown in Figures 8(d)-(f) The blue dotted lines are the simulation results with only applying the proposed multi-linear interpolation to calculate HTCs, and the red solid lines are obtained by simultaneously utilizing the HTC interpolation technique and considering the temperature-dependent effect of silicon thermal conductivity. The results show good tendency on predicting the temperature distribution of device layers by using the multi-linear interpolation techniques on calculating HTCs during the simulation procedure. Moreover, Figure 8(d) and Figure 8(e) demonstrate that the accuracy of predicted temperature distribution can be further improved by considering the influence of temperature dependence on thermal conductivity of silicon. Figure 8(f) shows that the effect of temperature induced thermal conductivity variation might be negligible in lower temperature values.

\subsection{Steady State Simulation}

To further demonstrate the proposed techniques, we also implement the STEAM model 
with three HTC correlation functions [9] [10] [11] used in STEAM to simulate these pseudo chips. Their errors compared with ours are shown in Table 1. "HTC interpol." represents to use the multi-linear interpolation technique for HTCs, and "HTC interpol. \& TDTCS" is to simultaneously use the interpolation technique for HTCs and consider the temperature-dependent effect on thermal conductivity. Both maximum errors and average errors for all three test chips by using the proposed techniques outperform the results by using the HTC correlation functions. Our average error can be less than $7.6 \%$, and even the maximum error is lower than $10.3 \%$ for all three test chips.

Finally, to demonstrate the robustness of the proposed method for 3-D ICs, a realistic 4-tier 3-D multiprocessor system-on-chip (3-D MPSoC) [19] is simulated. Its geometry structure is the same as Figure 1 and the total power consumption for each tier is $18.67 \mathrm{~W}$. The inlet temperature is $304.1 \mathrm{~K}$, and mass flux is $700 \mathrm{~kg} / \mathrm{m}^{2} \mathrm{~s}$. The error is compared with a commercial tool, ANSYS CFD. As shown in Table 1, our maximum error and average error are only $7.7 \%$ and $4.8 \%$, respectively. Although the runtime by using the HTC correlation functions is shorter than ours, their minimum average error is twice worse than ours. Hence, the amount of accuracy improvement is more than the previous three pseudo chips. This is a very promising result, since this 3-D MPSoC is a realistic design, and it means that the proposed techniques are robust for real designs.

\subsection{Transient Simulation}

The case is constant workload and simulation time is 0.2 second with power density $96.4 \mathrm{~W} / \mathrm{cm}^{2}$. Comparing with the ANSYS results, the maximum and average error of "HTC interpol." are about $12.6 \%$ and $7.8 \%$, and the maximum and average error of "HTC interpol. \& TDTCS" are about $10.7 \%$ and $7.3 \%$. It shows that considering the temperature-dependent effect on thermal conductivity is also important through these results and the similar trend with ANSYS result as well.

\section{Conclusion}

An effective and accurate thermal simulation method for two-phase liquid cooling 2-D/3-D ICs has been developed and implemented. The experimental results have demonstrated its accuracy improvement and effectiveness for the real designs.

Table 1. Error of Temperatures Compared with Thermal Sensors and ANSYS CFD.

\begin{tabular}{|c|c|c|c|c|c|c|c|c|c|c|c|c|c|c|c|}
\hline \multirow[t]{3}{*}{ Test case } & \multicolumn{9}{|c|}{ HTC correlation in STEAM } & \multicolumn{6}{|c|}{ Proposed } \\
\hline & \multicolumn{2}{|c|}{ Error (\%) } & \multirow{2}{*}{$\begin{array}{l}\text { Time } \\
(\mathrm{s})\end{array}$} & \multicolumn{2}{|c|}{ Error (\%) } & \multirow{2}{*}{$\begin{array}{l}\text { Time } \\
(\mathrm{s})\end{array}$} & \multicolumn{2}{|c|}{ Error (\%) } & \multirow{2}{*}{$\begin{array}{l}\text { Time } \\
(\mathrm{s})\end{array}$} & \multicolumn{2}{|c|}{ Error (\%) } & \multirow{2}{*}{$\begin{array}{l}\text { Time } \\
\text { (s) }\end{array}$} & \multicolumn{2}{|c|}{ Error (\%) } & \multirow{2}{*}{$\begin{array}{l}\text { Time } \\
\text { (s) }\end{array}$} \\
\hline & avg & $\max$ & & avg & $\max$ & & avg & $\max$ & & avg & $\max$ & & avg & $\max$ & \\
\hline Uniform & 10.9 & 15.6 & 29.4 & 23.3 & 27.8 & 29.1 & 10.1 & 12.9 & 29.3 & 7.6 & 10.3 & 33.2 & 6.7 & 9.7 & 35.4 \\
\hline 3-D MPSoC & 10.9 & 14.1 & 96.5 & 11.7 & 13.2 & 94.7 & 8.6 & 11.5 & 95.6 & 5.9 & 8.3 & 120.9 & 4.8 & 7.7 & 125.6 \\
\hline
\end{tabular}

$\dagger 3$-D MPSoC is compared with ANSYS CFD. 


\section{Acknowledgements}

The authors would like to thank Professor Suresh V. Garimella and Professor Stefan S. Bertsch for providing the measurement data. This work was supported in part by Ministry of Science and Technology of Taiwan under Grants MOST 105-2221-E-009-168, MediaTek Inc., and Industrial Technology Research Institute.

\section{References}

[1] Brunschwiler, T., Michel, B., Rothuizen, H., Kloter, U., Wunderle, B., Oppermann, H. and Reichl, H. (2009) Interlayer Cooling Potential in Vertically Integrated Packages. Microsystem Technologies, 15, 57-74. http://dx.doi.org/10.1007/s00542-008-0690-4

[2] Tuckerman, D.B. and Pease, R.F.W. (1981) High-Performance Heat Sinking for VLSI. Electron Device Letters, 2, 129-169.

[3] Mizunuma, H., Lu, Y.-C. and Yang, C.-L. (2011) Thermal Modeling and Analysis for 3-D ICs with Integrated Microchannel Cooling. IEEE Trans. Comput.-Aided Des. Integr. Circuits Syst., 30, 1293-1306. http://dx.doi.org/10.1109/TCAD.2011.2144596

[4] Sridhar, A., Vincenzi, A., Ruggiero, M., Brunschwiler, T. and Atienza, D. (2010) 3D-ICE: Fast Compact Transient Thermal Modeling for 3D ICs with Inter-Tier Liquid Cooling. Proc. Int. Conf. on Comput.- Aided Des., 463-470.

[5] Sridhar, A., Vincenzi, A., Ruggiero, M. and Atienza, A. (2012) Neural Network-Based Thermal Simulation of Integrated Circuits on GPUs. IEEE Trans. Comput.- Aided Des. Integr. Circuits Syst., 31, 23-36. http://dx.doi.org/10.1109/TCAD.2011.2174236

[6] Fourmigue, A., Beltrame, G., Nicolescu, G. and Aboulhamid, E.M. (2011) A Linear-Time Approach for the Transient Thermal Simulation of Liquid-Cooled 3-D ICs. Proc. Int. Conf. on Hardware/ Software Codesign and System Synthesis, 197-205. http://dx.doi.org/10.1145/2039370.2039402

[7] Fourmigue, A., Beltrame, G. and Nicolescu, G. (2013) Explicit Transient Thermal Simulation of Liquid-Cooled 3-D ICs. Proc. on Des. Autom. Test Eur. Conf., 1385-1390.

[8] Sridhar, A., Madhour, Y., Atienza, D., Brunschwiler, T. and Thome, J. (2013) STEAM: A Fast Compact Thermal Model for Two-Phase Cooling of Integrated Circuits. Proc. Int. Conf. on Comput.- Aided Des., 256-263. http://dx.doi.org/10.1109/iccad.2013.6691127

[9] Bertsch, S.S., Groll, E.A. and Garimella, S.V. (2009) A Composite Heat Transfer Correlation for Saturated Flow Boiling in Small Channels. Int. J. Heat and Mass Transfer, 52, 2110 2118. http://dx.doi.org/10.1016/j.ijheatmasstransfer.2008.10.022

[10] Tran, T.N., Wambsganss, M.W. and France, D.M. (1996) Small Circular- and RectangularChannel Boiling with Two Refrigerants. Int. J. Multiphase Flow, 22, 485-498. http://dx.doi.org/10.1016/0301-9322(96)00002-X

[11] Kandlikar, S.G. and Balasubramanian, P. (2004) Correlation to Transition, Laminar, and Deep Laminar Flows in Minichannels and Microchannels. Heat Transfer Engineering, 25, 86-93. http://dx.doi.org/10.1080/01457630490280425

[12] Demmel, J.W., Eisenstat, S.C., Gilbert, J.R., Li, X.S. and Liu, J.W. (1999) A Supernodal Approach to Sparse Partial Pivoting. SIAM J. Matrix Anal. Appl., 20, 720-755. http://dx.doi.org/10.1137/S0895479895291765

[13] Thome, J. Wolverine Heat Transfer Engineering Data Book III. http://www.wlv.com/products/databook/db3/DataBookIII.pdf

[14] Karypis, G. and Kumar, V. (2013) NIST Standard Reference Database. http://www.nist.gov/srd/nist23.cfm 
[15] Richard, F. and Nielson, G. (1980) Smooth Interpolation of Large Sets of Scattered Data. Int. J. Numerical Methods in Engineering, 15, 1691-1704.

http://dx.doi.org/10.1002/nme.1620151110

[16] Leturcq, P., Dorkel, J.M., Napieralski, A. and Lachiver, E.R.I.C. (1987) A New Approach to Thermal Analysis of Power Devices. IEEE Trans. Electron Devices, 34, 1147-1156. http://dx.doi.org/10.1109/T-ED.1987.23057

[17] Costa-Patry, E., Nebuloni, S., Olivier, J. and Thome, J.R. (2012) On-Chip Two-Phase Cooling with Refrigerant 85-Wide Multi-Microchannel Evaporator under Hot-Spot Conditions. IEEE Trans. Compon., Packag., Manuf. Technol., 2, 311-320.

[18] Cupelli, C., Lindemann, T., Moosmann, C., Niekrawietz, R., Glatzel, T., Litterst, C. and Koltay, P. (2008) Computational Fluid Dynamics (CFD) Software Tools for Microfluidic Applicationsa Case Study. IEEE Computers \& Fluids, 37, 218-235.

http://dx.doi.org/10.1016/j.compfluid.2007.07.014

[19] Sabry, M.M., Coskun, A.K., Atienza, D., Rosing, T.S. and Brunschwiler, T. (2011) EnergyEfficient Multiobjective Thermal Control for Liquid-Cooled 3-D Stacked Architectures. IEEE Trans. Comput.-Aided Des. Integr. Circuits Syst., 30, 1883-1896.

http://dx.doi.org/10.1109/TCAD.2011.2164540

\section{Submit or recommend next manuscript to SCIRP and we will provide best service} for you:

Accepting pre-submission inquiries through Email, Facebook, LinkedIn, Twitter, etc. A wide selection of journals (inclusive of 9 subjects, more than 200 journals)

Providing 24-hour high-quality service

User-friendly online submission system

Fair and swift peer-review system

Efficient typesetting and proofreading procedure

Display of the result of downloads and visits, as well as the number of cited articles Maximum dissemination of your research work

Submit your manuscript at: http://papersubmission.scirp.org/

Or contact jcc@scirp.org 